\title{
New requirements on beach design: limiting states condition
}

\author{
J. C. Santás \& J. M. de la Peña \\ Centro de Estudios de Puertos y Costas (Harbours and Coasts Studies \\ Centre), CEDEX, Spain
}

\begin{abstract}
The natural evolution of a beach follows a sequence of different situations: accretion, erosion, annual oscillations and stable phases, but the beach is not an isolated coastal unit and interacts with its surrounding areas. Problems appear when its evolution affects other entities on the coastal zone related to infrastructures or leisure use or to the territorial development of the coastal region.

Problems also brought about by urban pressure and the natural tendency to invade and use the coastal zone has led to designing the coastal edge without taking into account the effects of bad weather states or storms, as it is for other coastal structures. When this happens and damage occurs, public opinion establishes a relation of damage with other circumstances or factors different to the real question, which is that the beach design has not taken into account extreme conditions: the land development has considered the beach only in its best or medium weather conditions.

This paper presents new criteria to designing beaches taking into account its state of maximum sea impact, that is, its limit state.
\end{abstract}

Keywords: beach design, stormy boundary conditions for beach design.

\section{Introduction}

Often beaches, which have not had problems throughout their history, suddenly appear to hatch an unusual situation, sometimes due to coastal erosion, but at other times because the beach is considered as a static unit in its best state while the worst climate conditions were considered for other coastal systems. This led to designing coast without taking into account the evolution that may happen in 
storm conditions. When these climatic periods appear, public opinion tends to be based around a belief that the beach has always been in a good condition, without considering that it was designed based on those good conditions. Comments like 'The beach has disappeared' or 'These are the consequences of the climate change that is coming!' are regularly expressed. It does not mean that the mean level variation does not exist, but there are other factors to be taken into account. The most important factor is due to the coastal land uses development or Coastal Zone Development (CZD), which has changed for new uses and citizen demands. It has conditioned the beach, its evolution and its own state. Figure 1 shows the coastal historic evolution of the Spanish area between Gerona to Blanes on the Spanish Mediterranean coast in the Catalunia Region.

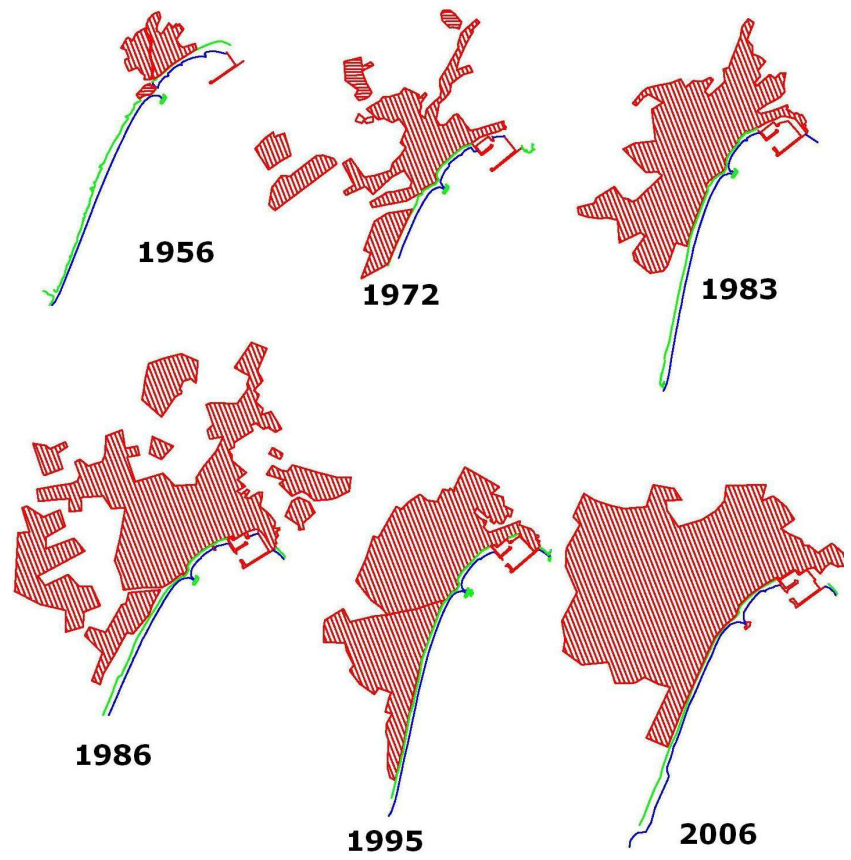

Figure 1: Coastal evolution of Gerona to Blanes coast.

\section{Current concept of 'beach limit'}

The basics to designing a beach, if it is in an urban area, are managed by the following aspects:

-The beach is in equilibrium when it is in its summer state, summer profile.

-The beach width has to be designed in view of the public use. Some designers consider that this may be the line where the vegetation begins or ought to begin.

These considerations have created problems when the beach is included in an urban domain. In some cases these assumptions have produced breaks on promenades and loss of foundations of the nearest buildings, figure 2 . 


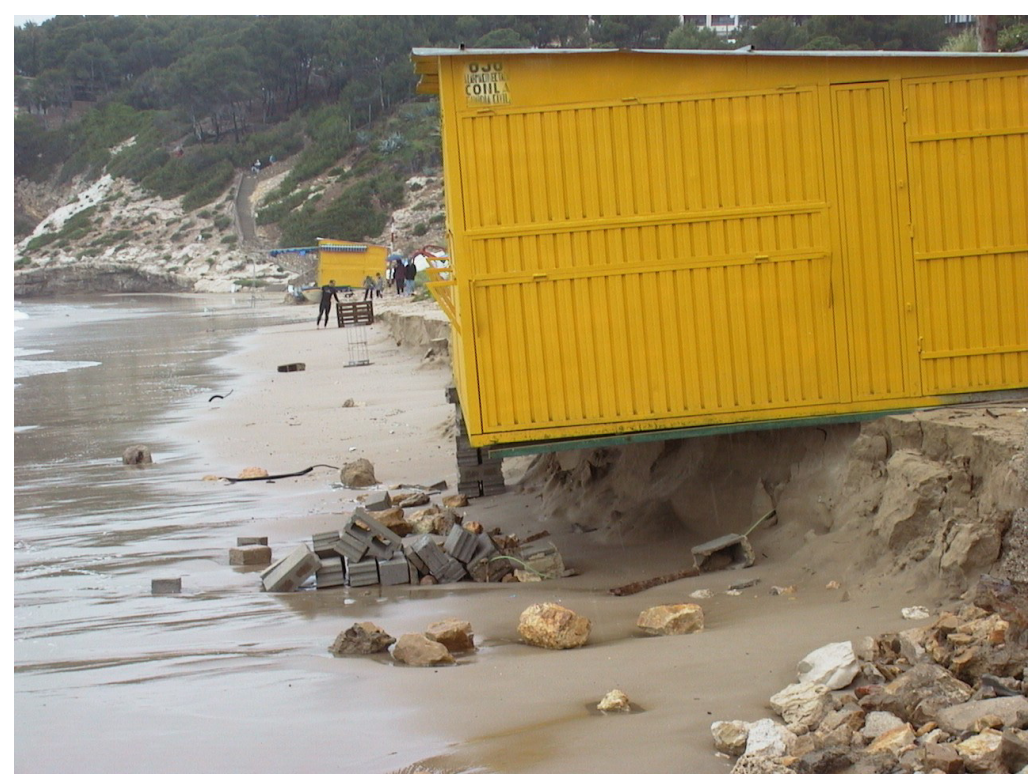

Figure 2: Loss of foundation of temporal stands located on a beach due to a storm.

This last concept creates problems because there are three functions of a beach: as coastal defence, as a place for public use and recreation, and as a biological habitat. On the other hand, it is focussed on a static beach without external actions that are inevitably going to modify it. The littoral dynamic is continuously acting on it and the beach width has to be compatible with the evolution forced by these external factors.

The beach is a dynamic element as real as the forces that acts upon it and it has a very high energetic dissipation capacity. It seems that the design should considerer all the external forces in its 'maximum state' as it does for other civil coastal structures, and that consideration should be made for its future in 50 or 100 years time. These concepts dealing with the natural states of a beach are going to be introduced on the Spanish Coasts Authority Recommendations (Peña and Sánchez [1]).

Other questions that need to be included are about the uncertainties that aspects like beach equilibrium profile and sea waves climate have (SánchezArcilla [2]).

In this line the idea of 'beach extreme states' would accept also that these are bands instead of lines. The equilibrium profile would be the centre line that represents the 'medium conditions' and there were a fork, whose width ought to be related to return period of waves and meteorological actions, including sea level change. All these sea actions and their effect need to be well defined from field data. 


\section{Minimum width of a beach}

The dry beach width has to be enough in all the circumstances in the way of defence and protect such infrastructures and the environmental values located behind the beach. The first question to define is the time for which this beach width has to be maintained, the beach width return period $A_{1}$.

This minimum value of beach width has to be a value to ensure the minimum needed to protect structures and goods, and values of the beach, taking into consideration the total amount of all the predicted losses of width, permanent, progressive or time dependent.

These individual losses are:

- A1: erosion, defined as the loss of beach width for the return period

- A2: annual oscillation defined by the tilt summer-winter on the profile, evaluated from the maximum distance waited into the return period.

- A3: width variation by sea level increase due to the maximum annual increase due to other factors (run-up, meteorological and tidal elevations) to be waited into the return period

- A4: backup width to ensure the roll of the beach as defender of goods.

- A5: width variation caused by sea level increase due to the climatic change along the estimated return period.

So the total width change could be estimated as $A 1=\sum A_{i}(i=1$ to 5$)$.

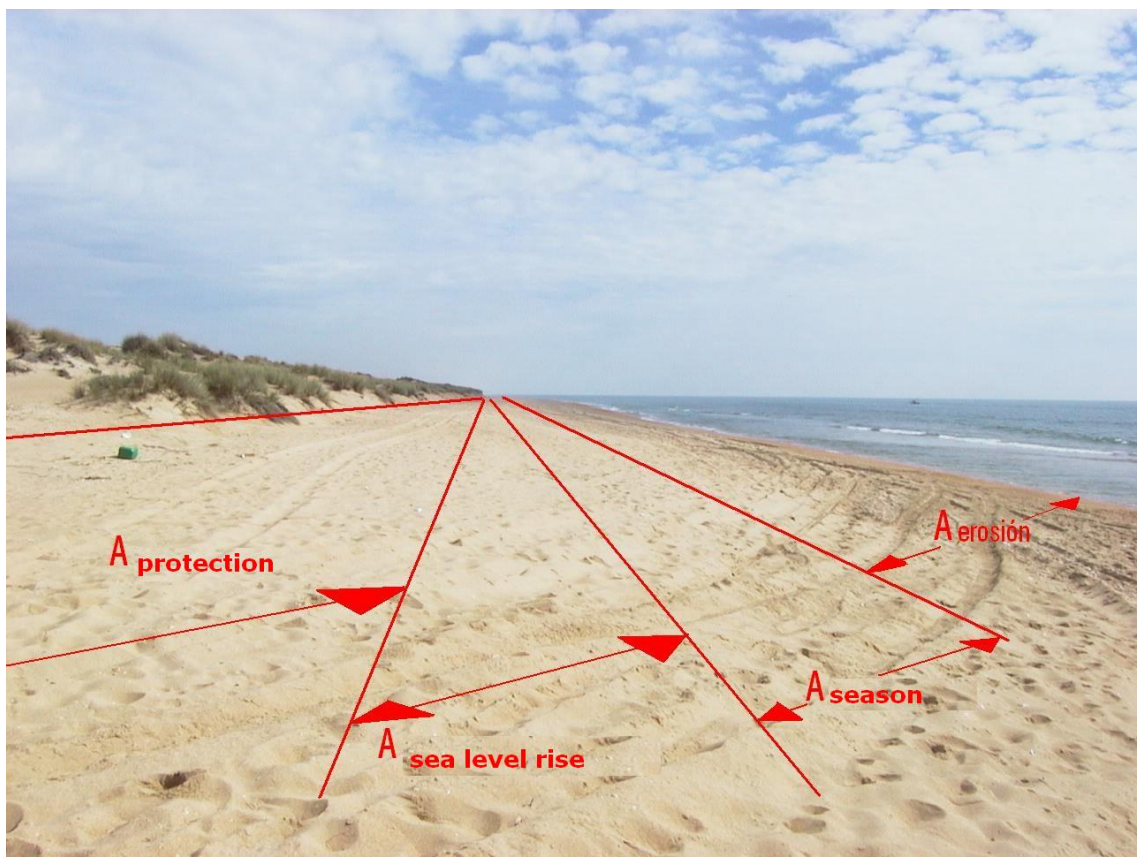

Figure 3: Different components for the beach width. 
Only two of the upper individual considered aspects, A1 (annual oscillation) and A3 (tilting due to run-up, meteorological, astronomical and waves actions) have to be estimated from extreme climate conditions into the return period. These are those that configure the limiting states of the beach.

\section{Limiting states of the beach}

The limiting states of the beach are two virtual lines: the upper dry beach line and the real closure depth line. The beach is defined between these two limits but they are not fixed lines. The deepest line is the real limit for all kind of movement of the sedimentary layer that is defined by the sea states (wave actions and tide) and the characteristics of sediment. For the dry limit the definition is very complex as we have shown before. To prevent extra actions coming from outside the beach that affect it, a backup distance for both sides has to be added: backup for dry beach and backup for submerged limit.

The closure depth, or limit for sediment motion, is defined by 2 ways: 'active depth', $\mathrm{d}_{\mathrm{i}}$, as the limit for transversal and longitudinal motion of sediment obtained from sea waves and tide characteristics (Birkemeier [3]), formula or other similar expressions, and the deeper limit, $\mathrm{d}_{1}$, for all kind of sediment motions, given, as an example, by the CERC's [4] formula as:

$$
d_{i}=3,5 \cdot H_{s, 0.137}
$$

that give us the deepest limit obtained on the bibliography, function of the significant wave height that shows the $\mathrm{p}=1-0.137$ probability (approximately: 12 hours in a year) on average distribution of waves. For this limit all the profiles obtained coincide. Figure 4 shows the different parts of profile, as Hallermeier [5].

The maximum depth and the active depth are defined in terms of Hs, but it would be in relation to the return period. It is: by the storm regime instead of medium regime of Hs. In the same way the upper limit has to be defined by the maximum level reached by waves in storm conditions and the profile of the dry beach that is related to the initial state of the beach, the grain size and the slope.

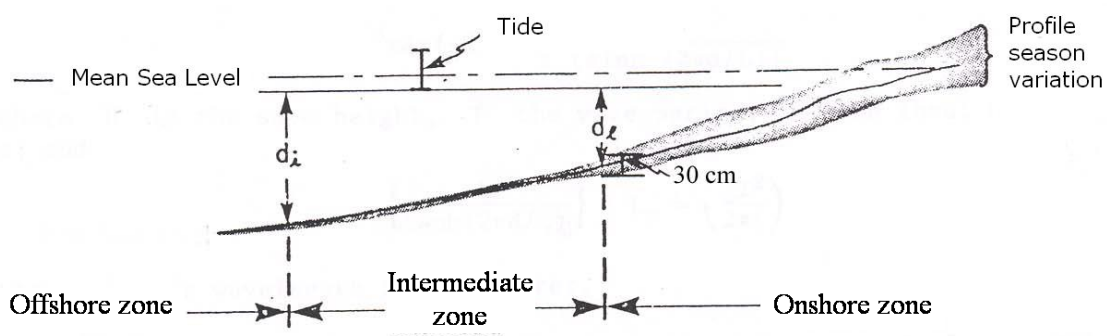

Figure 4: Layout of the beach profile and summer-winter oscillation. 
Starting from an equilibrium profile the beach answers to the energy of an incident storm by a change in its profile as well as moving material from the dry part to the submerged part of the profile. Usually submerged bars appear to diminish the waves' energy by turbulence and movement of sediment. This action also modifies the submerged part of the profile with less slope and fine sediment in comparison to the shore line where the beach increased the grain size and slope.

Before the surf zone, waves coming by very shallow water show an elevation of the mean sea level, it is the set-up that is a function of the significant height $\mathrm{H}_{\mathrm{s} 0}$ on depth water. After the breaking zone the waves go up by the beach slope until its final stop by losing all the energy, returning the water layer to the sea. The maximum point reached by water gives the run-up level.

The study of what is the maximum level reached by the water has to be done after knowing the sea waves climate. Maximum levels for the set-up and the runup are determined in function of the characteristics of the incident sea waves (direction, height and period) as well as the beach slope.

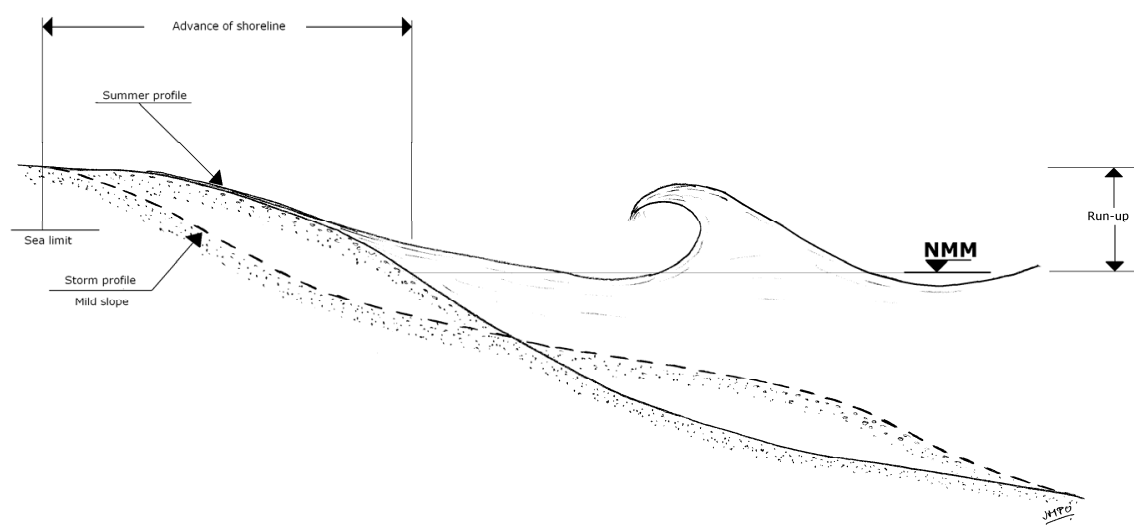

Figure 5: $\quad$ Summer and winter profile, run-up and maximum elongation over the beach for storm.

\section{Beach limit from the point of view of Coastal Zone Development}

Following the previous described aspects, the design of an urban beach has to ensure an appropriate width as well as the assumption of the natural dynamic conditions and the natural evolution of the beach, for erosion and sea level displacement due to the climatic change.

Figure 6 shows a clear case where the beach could have not been designed for citizen uses. It is the 'La Concha' beach, San Sebastián, Vasc Country, Spain, located $200 \mathrm{~m}$ westward from Zurriola beach. The city invaded the beach area three centuries ago and now it is not possible to fix the situation. 
The equilibrium between these points affects the Coastal Zone Development, mainly to the space located between the urban area and the beach. In the Spanish case, the Coastal Authorities, Dirección General de Sostenibilidad de la Costa y del Mar, has given a regulation that analyse the $\mathrm{CZ}$ from three points of view:

a. Transformation of marine urban facades.

b. Treatment of walking ways

c. Access, transit and public use of the coast.
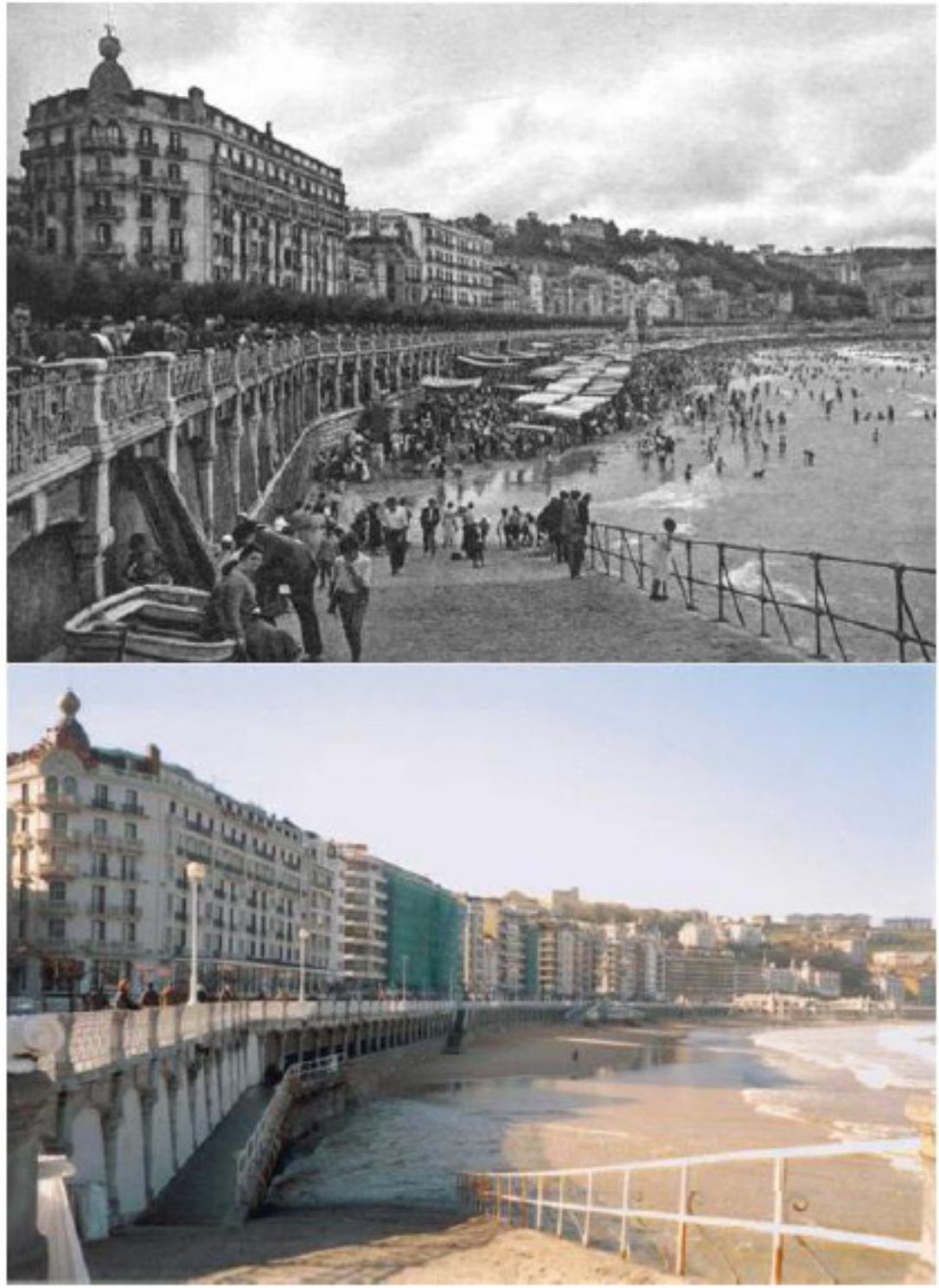

Figure 6: 'La Concha' beach. High spring tide and low spring tide. 


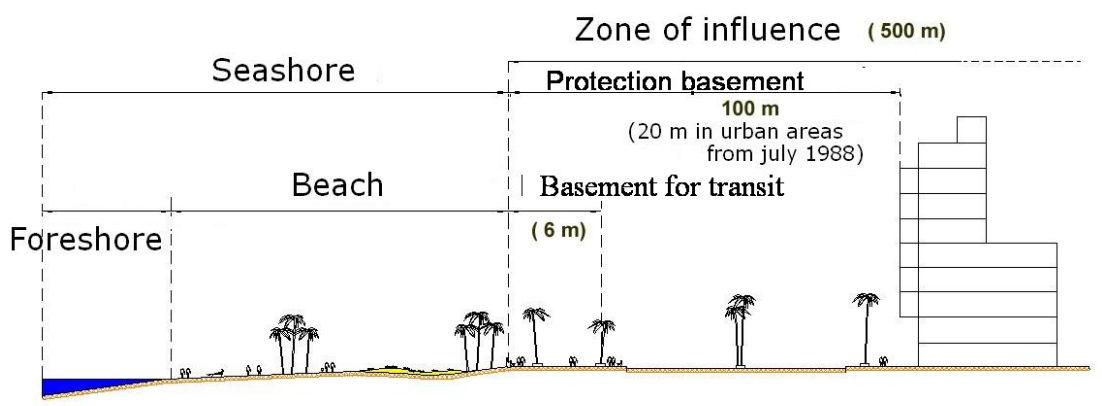

Figure 7: Coastal Zone regulation, Law 22/1988, Environment Spanish Ministry (Peña and Sánchez [1]).

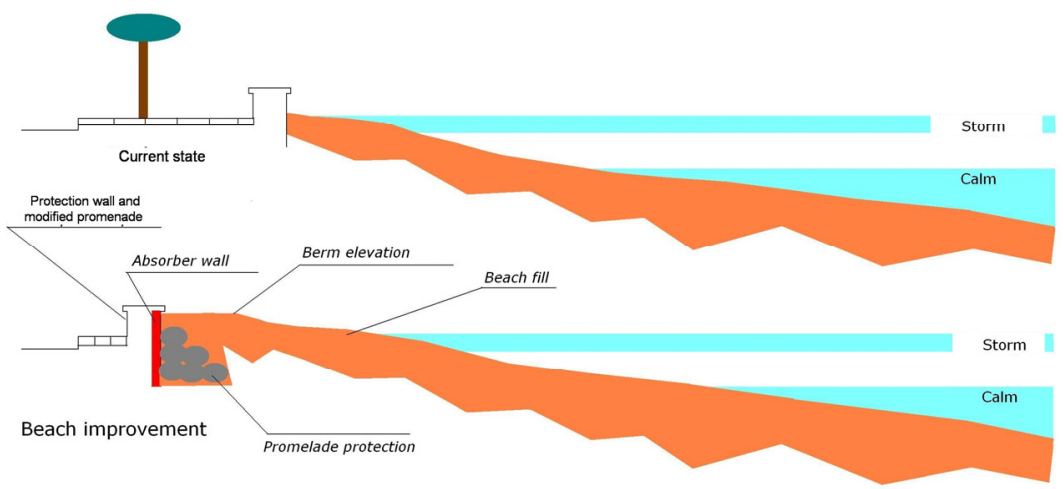

Figure 8: Transformation of a maritime front to allow free movement of the beach.

The limits that have to be considered for the regulation of a beach are the maximum elevation of sea level for a period of time equal or greater than the return period of a littoral urban structure, a walking promenade for example, after the addition of a backup margin due to the uncertainties of the data. This margin could be in the order of $30 \%$.

Also, it needs to be taken into account that the beaches are going to be an area for citizens' use, with growing use in the summer time owing to more visits by its population.

\section{Conclusions}

1) It is not possible to maintain the current parameters used for the beach design overall for urban beaches, as well as their relationship to the Coastal Zone Development. These parameters are: the equilibrium profile adding a supplementary width that is defined for the citizen uses, because this concept has drive us to big problems and failures on the coastal zone of coastal cities. 
2) The state of maxima sea actions (waves, tides, winds and currents) has to be considered for the beach limits and beach design in relation to the coastal border. For that a return period has to be used never less than the life period of the adjacent urban developments, typically between 50 and 100 years.

3) Due to the uncertainties of data and especially for the maximum elevation values of sea level, an error band ought to be taken into account. If not able to be accurately predicted based on available data then a confidence band of $30 \%$ could be included as a safety parameter.

\section{References}

[1] Peña, J.M. and Sánchez, V., Asistencia técnica para la redacción de dos instrucciones para la Dirección General de Costas;(in Spanish) Centro de Estudios de Puertos y Costas, CEDEX(report n 22-408-5-001 for D.G. de Costas), 2008

[2] Sánchez-Arcilla, A.; Personal communication (20 $0^{\text {th }}$-April-2009), Head of Engineering Laboratotium, LIM; Civil Engineering School, Politechnical University of Barcelona. Spain.

[3] W.A. Birkemeier, Field data on seaward limit of profile change, Journal of W.,P.,C. and Oc. Eng., A.S.C.E., vol 111, 3; pp. 598 to 602., 1985.

[4] CERC, 1998, Coastal Engineering Research Centre, Engineering and design- Coastal Eng. Manual, Part III, Cross Shore sediment transport processes; Dep. of the Army, US Corps of Eng.. Pub.: 1110-292.

[5] Hallermeier, R.J. (1978). /Uses for Calculated Limit Depth to Beach Erosion/; Proceedings of 16th International Conference on Coastal Engineering (pp. 1493 a 1512) 